\title{
Long-range orbital hybridization in remote epitaxy: the nucleation mechanism of GaN on different substrates via single layer graphene
}

Yipu $\mathrm{Qu}^{1}, \mathrm{Yu} \mathrm{Xu}^{* 1,2}$, Bing Cao*3,4, Yuning Wang ${ }^{1}$, Jianfeng Wang ${ }^{1,2}$, Lin $\mathrm{Shi}^{5}$ and $\mathrm{Ke}$ $\mathrm{Xu}^{* 1,2}$

1. Suzhou Institute of Nano-Tech and Nano-Bionics (SINANO), Chinese Academy of Sciences (CAS), Suzhou 215123, People's Republic of China

2. Suzhou Nanowin Science and Technology Co., Ltd., Suzhou 215123, People's Republic of China

3. School of Optoelectronic Science and Engineering \& Collaborative Innovation Center of Suzhou Nano Science and Technology, Soochow University, Suzhou 215006, People's Republic of China

4. Key Lab of Advanced Optical Manufacturing Technologies of Jiangsu Province \& Key Lab of Modern Optical Technologies of Education Ministry of China, Soochow University, Suzhou 215006, People's Republic of China

5. School of Materials Science and Engineering, Yancheng Institute of Technology, Yancheng 224051, People's Republic of China

*Corresponding author e-mail: yxu2007@sinano.ac.cn (Yu Xu); bcao2006@163.com (Bing Cao); kxu2006@sinano.ac.cn (Ke Xu). 


\section{Experiment 1}

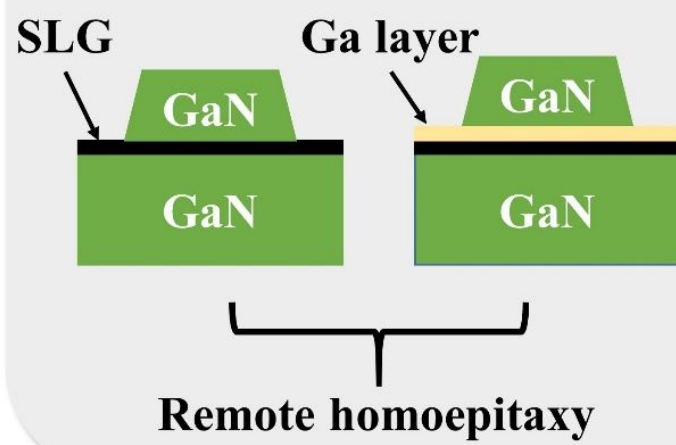

Experiment 2

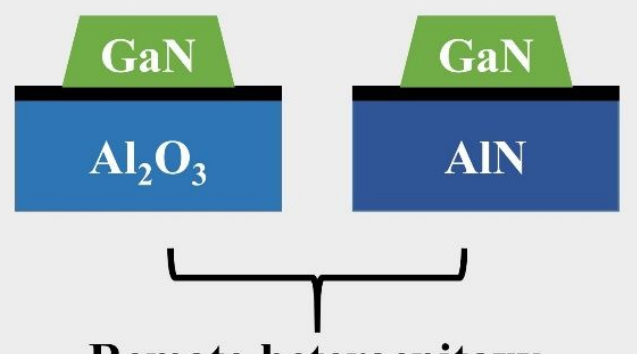

Remote heteroepitaxy

Figure S1. Schematic diagram of the growth of two sets of experiments with remote homo- and heterepitaxy.

(a)

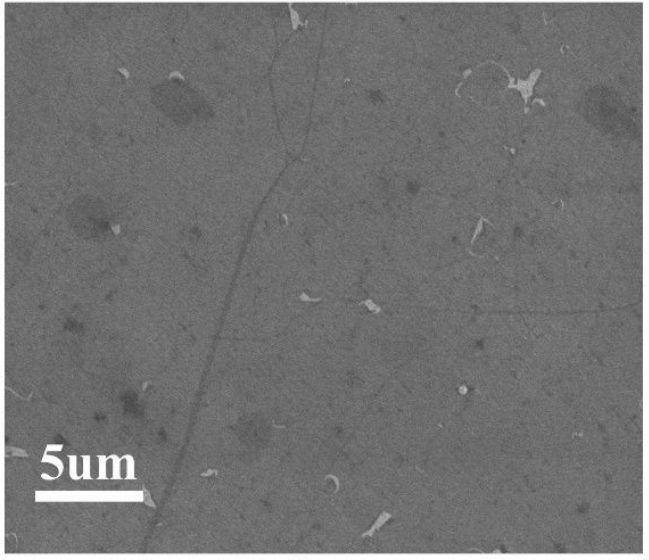

(c)

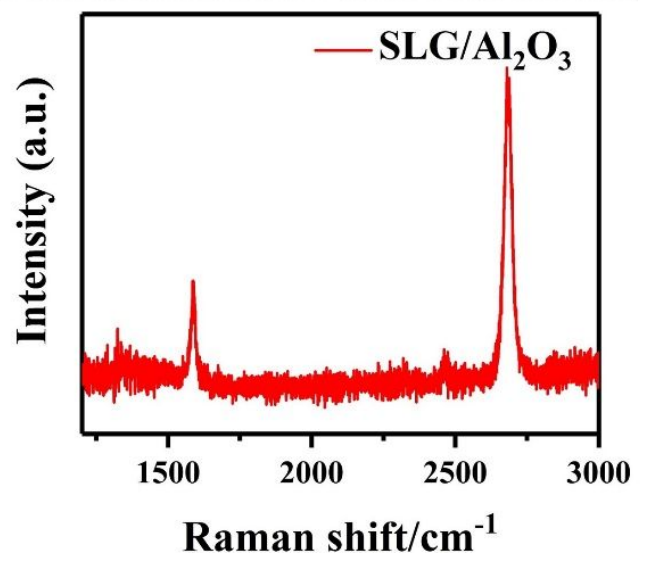

(b)

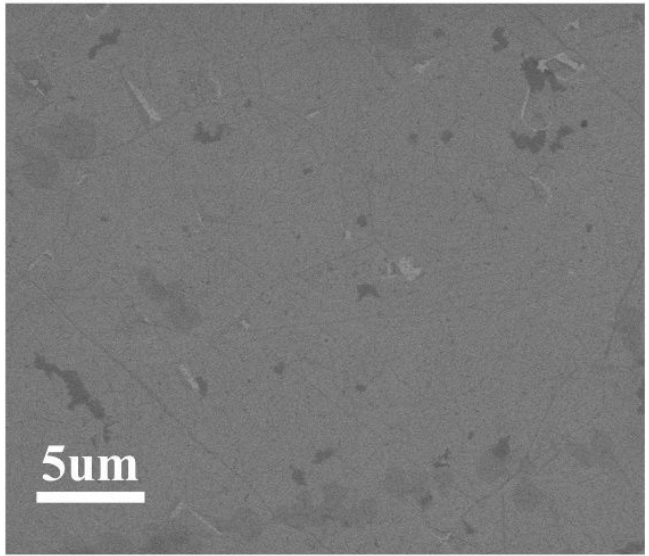

(d)

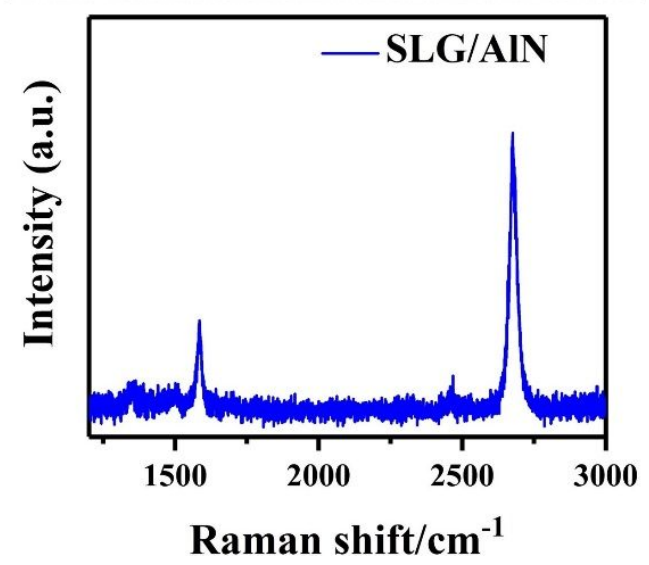

Figure S2. (a)(b) SEM images of SLG transferred on $\mathrm{Al}_{2} \mathrm{O}_{3}$ and $\mathrm{AlN}$ substrates, respectively. (c)(d) Raman spectra of SLG transferred on $\mathrm{Al}_{2} \mathrm{O}_{3}$ and AlN substrates, respectively. 
(a)

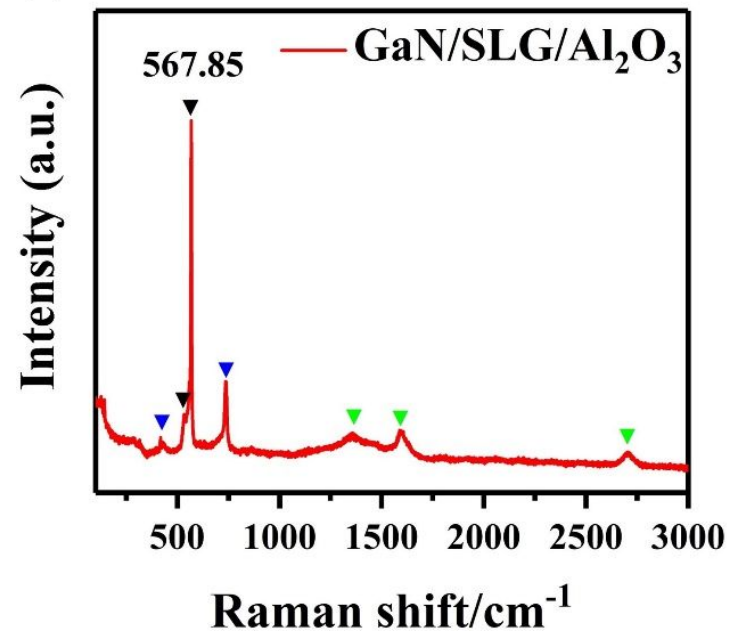

(b)

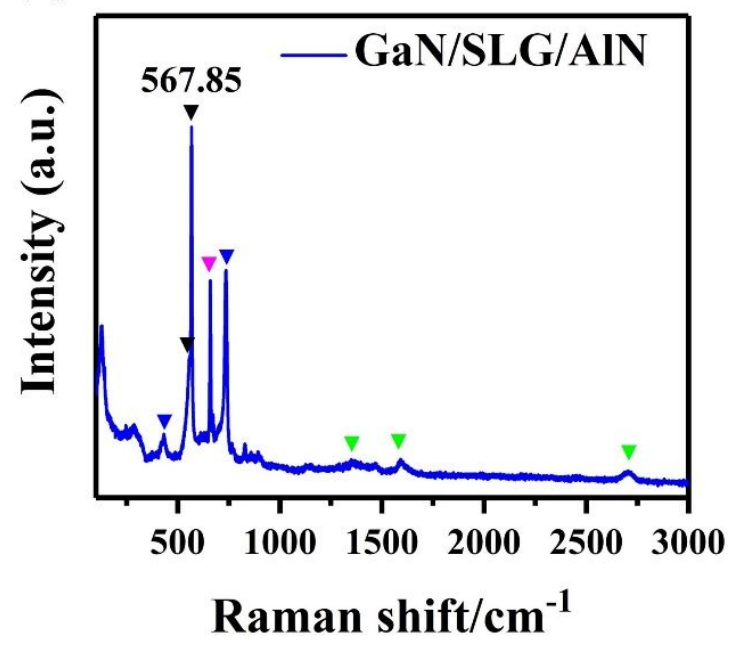

Figure S3. Raman spectra of GaN NLs grown on (a) $\mathrm{SLG} / \mathrm{Al}_{2} \mathrm{O}_{3}$ and (b)

SLG/AlN substrates, respectively.
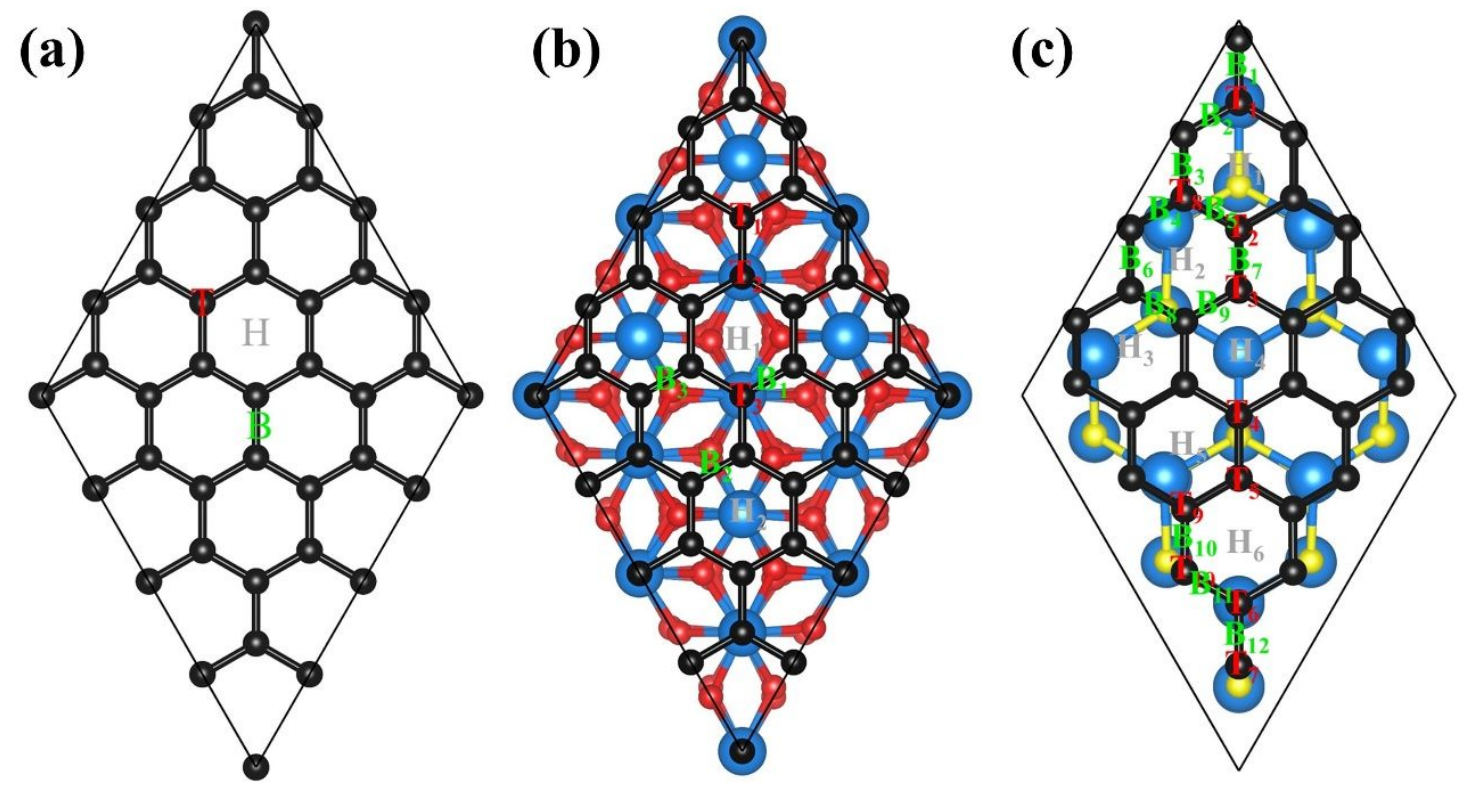

Figure S4. All classical adsorption sites ( $\mathrm{T}, \mathrm{H}$ and $\mathrm{B}$ ) for $\mathrm{Ga}$ and $\mathrm{N}$ atom adsorption on three substrates (a) SLG (b) $\mathrm{SLG} / \mathrm{Al}_{2} \mathrm{O}_{3}$ (c) SLG/AIN. 


\begin{tabular}{|c|c|c|c|}
\hline \multirow{4}{*}{ SLG } & Site & Ga & $\mathbf{N}$ \\
\hline & $\mathbf{T}$ & -0.923 & -2.479 \\
\hline & $\mathbf{H}$ & -0.933 & -0.668 \\
\hline & B & -0.910 & -3.848 \\
\hline \multirow{8}{*}{$\mathrm{SLG} / \mathrm{Al}_{2} \mathrm{O}_{3}$} & $T_{1}$ & -1.166 & -3.152 \\
\hline & $\mathbf{T}_{2}$ & -1.190 & -2.472 \\
\hline & $T_{3}$ & -1.121 & -2.979 \\
\hline & $\mathbf{H}_{1}$ & -1.162 & -0.884 \\
\hline & $\mathbf{H}_{2}$ & -1.199 & -0.850 \\
\hline & $\mathbf{B}_{1}$ & -0.808 & -3.889 \\
\hline & $\mathbf{B}_{2}$ & -0.815 & -3.897 \\
\hline & $\mathbf{B}_{3}$ & -0.805 & -3.916 \\
\hline \multirow{28}{*}{ SLG/AIN } & $T_{1}$ & -1.727 & -4.882 \\
\hline & $\mathbf{T}_{2}$ & -1.569 & -5.367 \\
\hline & $\mathbf{T}_{3}$ & -1.456 & -4.315 \\
\hline & $\mathbf{T}_{4}$ & -1.447 & -4.648 \\
\hline & $T_{5}$ & -1.469 & -6.591 \\
\hline & $T_{6}$ & -1.774 & -4.212 \\
\hline & $\mathbf{T}_{7}$ & -1.496 & -4.734 \\
\hline & $\mathbf{T}_{8}$ & -1.578 & -4.887 \\
\hline & $T_{9}$ & -1.532 & -5.041 \\
\hline & $\mathbf{T}_{10}$ & -1.462 & -6.384 \\
\hline & $\mathbf{H}_{1}$ & -2.672 & -4.529 \\
\hline & $\mathbf{H}_{2}$ & -1.415 & -3.696 \\
\hline & $\mathbf{H}_{3}$ & -1.409 & -3.404 \\
\hline & $\mathbf{H}_{4}$ & -1.338 & -3.113 \\
\hline & $\mathbf{H}_{5}$ & -1.355 & -3.589 \\
\hline & $\mathbf{H}_{6}$ & -1.471 & -4.253 \\
\hline & $\mathbf{B}_{1}$ & -1.500 & -5.362 \\
\hline & $\mathbf{B}_{2}$ & -1.341 & -5.571 \\
\hline & $\mathbf{B}_{3}$ & -1.416 & -5.542 \\
\hline & $\mathbf{B}_{4}$ & -1.335 & -4.612 \\
\hline & $\mathbf{B}_{5}$ & -1.522 & -5.287 \\
\hline & $\mathbf{B}_{6}$ & -1.437 & -5.376 \\
\hline & $\mathbf{B}_{7}$ & -1.366 & -5.194 \\
\hline & $\mathbf{B}_{8}$ & -1.512 & -5.377 \\
\hline & $\mathbf{B}_{9}$ & -1.357 & -5.136 \\
\hline & $\mathbf{B}_{10}$ & -1.431 & -5.627 \\
\hline & $\mathbf{B}_{11}$ & -1.519 & -5.249 \\
\hline & $\mathbf{B}_{12}$ & -1.307 & -5.101 \\
\hline
\end{tabular}


Table S1. Adsorption energies of the various adsorption sites labeled in

S4.

(a)
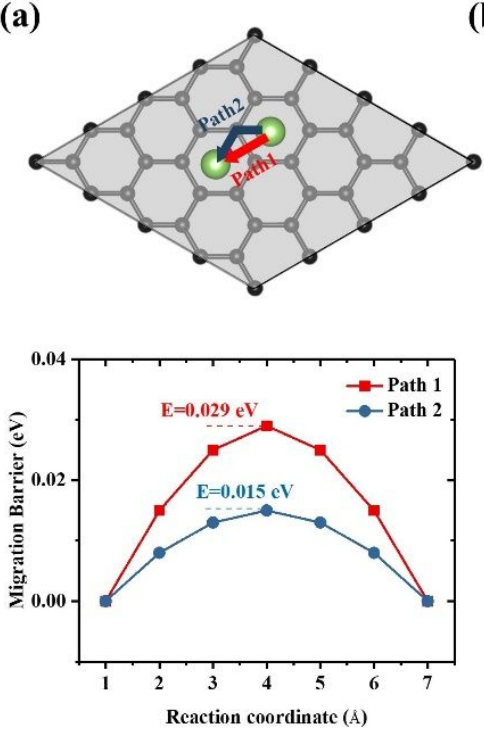

(b)
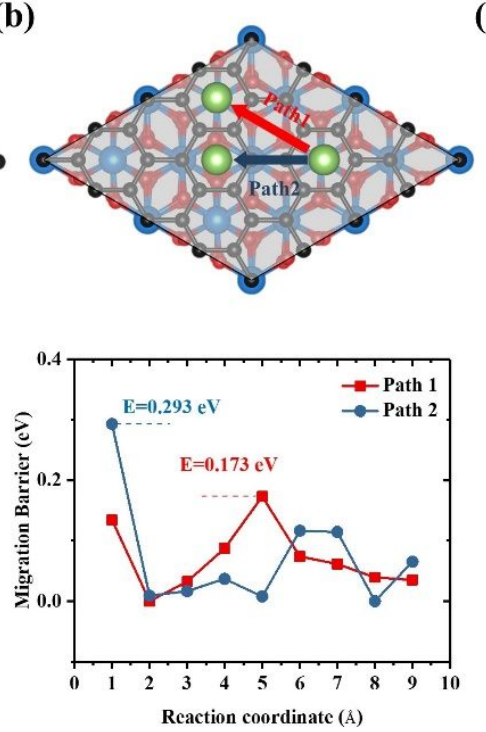

c)
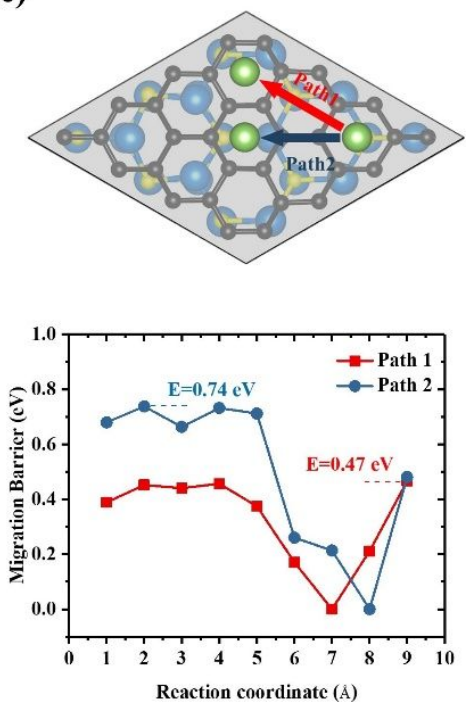

Figure S5. Migration energies of Ga atoms on different substrates. (a)

Ga on SLG. (b) Ga on $\mathrm{SLG} / \mathrm{Al}_{2} \mathrm{O}_{3}$ (c) Ga on SLG/AIN. 

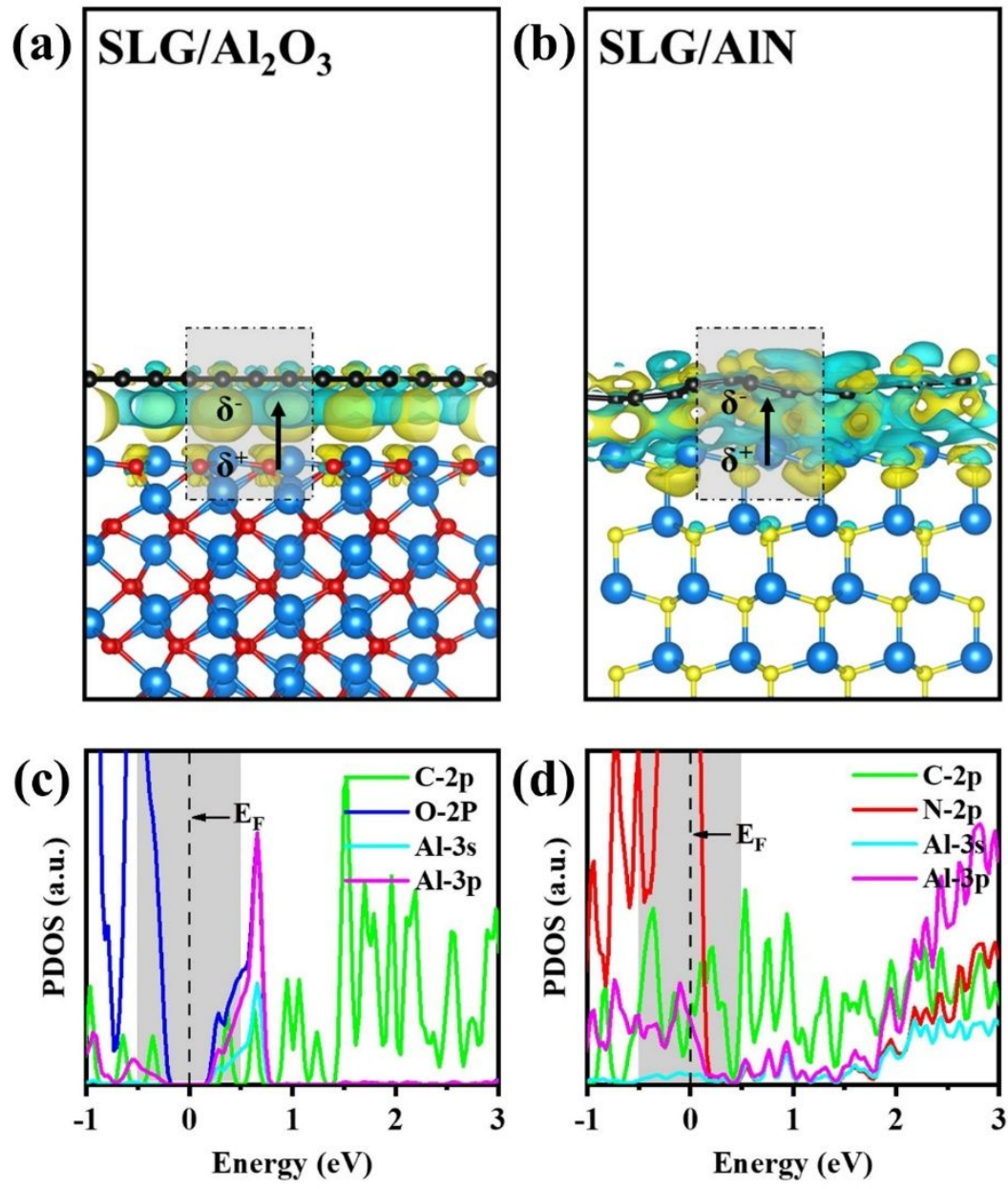

Figure S6. CDD of (a) SLG/ $\mathrm{Al}_{2} \mathrm{O}_{3}$ and (b) SLG/AlN systems. PDOS of (c) $\mathrm{SLG} / \mathrm{Al}_{2} \mathrm{O}_{3}$ and (d) SLG/AIN systems.

\begin{tabular}{cccc}
\hline & $\mathbf{Q}_{\text {sub }}$ & $\mathbf{Q}_{\text {SLG }}$ & $\mathbf{Q}_{\text {Ga }}$ \\
\hline SLG/ $\mathbf{A l}_{\mathbf{2}} \mathbf{O}_{\mathbf{3}}$ & -0.336 & 0.336 & -- \\
SLG/AIN & 0.676 & -0.676 & \\
$\mathbf{G a} / \mathbf{S L G}$ & -- & -0.308 & $\mathbf{0 . 3 0 8}$ \\
$\mathbf{G a} / \mathbf{S L G} / \mathbf{A l}_{\mathbf{2}} \mathbf{O}_{\mathbf{3}}$ & -0.459 & 0.137 & $\mathbf{0 . 3 2 2}$ \\
$\mathbf{G a} / \mathbf{S L G} / \mathbf{A l N}$ & 0.202 & -0.707 & $\mathbf{0 . 5 0 5}$ \\
\hline
\end{tabular}

Table S2. Bader charge transfer analysis of Ga atom adsorbed on SLG, $\mathrm{SLG} / \mathrm{Al}_{2} \mathrm{O}_{3}$ and $\mathrm{SLG} / \mathrm{AlN}$ three substrates, respectively. 


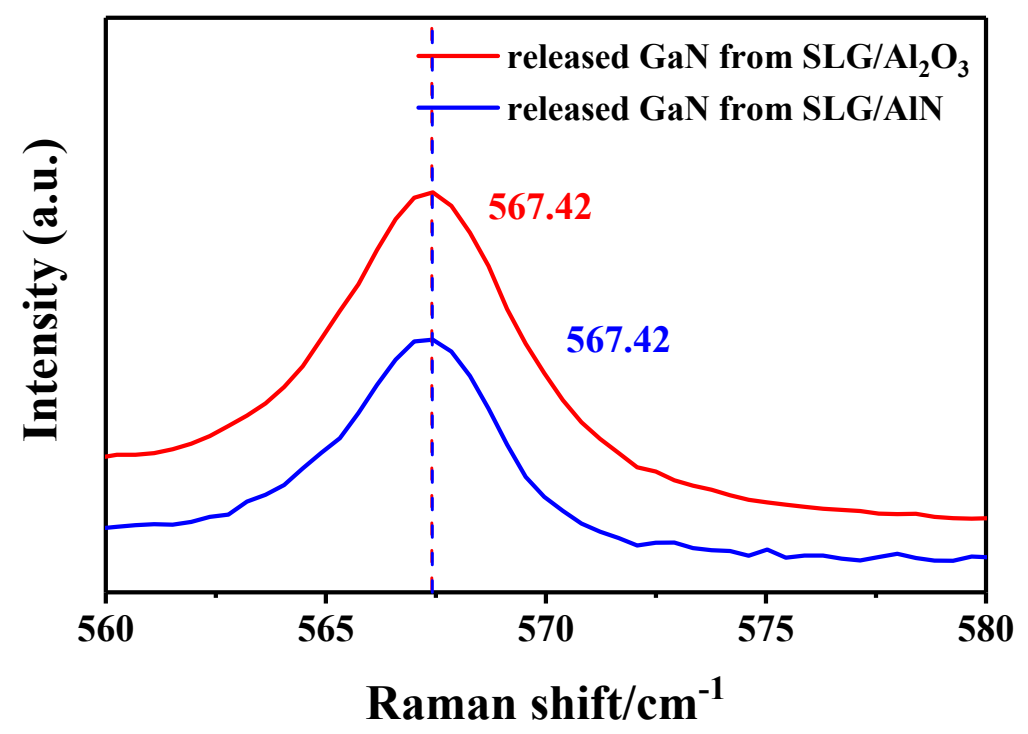

Figure S7. Raman spectra of GaN NLs backside after stripping from $\mathrm{SLG} / \mathrm{Al}_{2} \mathrm{O}_{3}$ and $\mathrm{SLG} / \mathrm{AlN}$ surfaces using conductive adhesive, respectively. 